\title{
Polnische und deutsche Phraseologismen mit den Komponenten kot/Katze und pies/Hund im "Słownik języka polskiego" von Samuel Bogumił Linde und im "Deutschen Wörterbuch" von Jacob und Wilhelm Grimm
}

\author{
Dominika Janus (Danzig)
}

\begin{abstract}
The aim of the article is to analyze Polish and German idioms containing names of animals, namely cat and dog in Słownik języka polskiego by Samuel Bogumił Linde and Deutsches Wörterbuch by Jacob and Wilhelm Grimm. Firstly, it is to be stated how many of those idioms mentioned in both of the dictionaries have survived and how many are extinct. The meanings, symbols and images hidden in the idioms regarded as the historical ones seem to be particularly interesting. The last point of the analysis refers to the fact of how much Polish and German idioms containing the same names of animals are similar.
\end{abstract}

\section{$1 \quad$ Einleitung}

Das Ziel meines Beitrags ist eine Analyse der polnischen und deutschen Phraseologismen im "Słownik języka polskiego" von Samuel Bogumił Linde und im "Deutschen Wörterbuch" von Jacob und Wilhelm Grimm. Die beiden Nachschlagewerke sind in einem vergleichbaren Zeitraum entstanden. Die Analyse der in ihnen verzeichneten festen Wortverbindungen soll einen Beitrag zur polnischen und deutschen historischen Phraseographie leisten.

Das "Słownik języka polskiego" (SJP) von Samuel Bogumił Linde erschien in den Jahren 1807-1814 und umfasst sechs Bände. Das SJP, das erste wissenschaftliche Wörterbuch der polnischen Sprache, in dem der Wortschatz aus den Jahren 1550-1800 verzeichnet ist, wird als ein bahnbrechendes und epochales Werk in der Geschichte der polnischen Lexikographie bezeichnet (vgl. Matuszczyk 2006: 13). Das "Deutsche Wörterbuch" (DWB) von Jacob und Wilhelm Grimm, das aus 16 Bänden in 32 Teilbänden besteht, wurde in den Jahren 18541961 veröffentlicht (der erste Band erschien zwar in der Mitte des 19. Jahrhunderts, das Wörterbuch wurde aber erst 1961, lange nach dem Tod seiner Autoren, beendet). Im DWB wird die Entwicklung des Wortschatzes seit seinen Anfängen im Althochdeutschen nachgezeichnet. Schlaefer präzisiert:

Das Grimmsche Wörterbuch [...] deckt zwar in der Stichwortaufnahme den neuhochdeutschen Wortschatz ab $1450 \mathrm{ab}$, beschreibt jedoch den Gebrauch der gebuchten Wörter sprachperiodenübergreifend (panchronisch) vom Althochdeutschen bis zum jeweiligen Redaktionszeitpunkt, so dass eine sprachstufenbezogene neuhochdeutsche Betrachtungsweise weitgehend neutralisiert wird.

(Schlaefer 2002: 118)

Linguistik online 74, 5/15 - http://dx.doi.org/10.13092/lo.74.2225

CC by 3.0 
Sowohl das SJP, als auch das DWB zählen unumstritten zu den bedeutendsten Leistungen der polnischen bzw. deutschen Lexikographie. Obwohl die beiden historisch orientierten Wörterbücher allgemeinen Charakter haben, bilden sie in großer Zahl den phraseologischen Bestand ab. Das SJP sowie das DWB als Belegwörterbücher listen zu den einzelnen Lemmata und oft auch zu den Phraseologismen authentische Sprachbelege auf, die das tatsächliche Vorkommen der festen Wortverbindungen beweisen (vgl. Pepłowski 1970: 108; Dräger 2009: 39).

Da sich sowohl im SJP als auch im DWB Phraseologisches ohne übersichtliche Anordnung unter den einzelnen Lemmata befindet, müssen die Phraseologismen aus den Wörterbüchern erst exzerpiert werden, was oft nicht einfach ist (zur Identifikation von Phraseologismen in älteren Texten, darunter auch Wörterbüchern, vgl. Burger/Buhofer/Sialm 1982: 346-382). Im DWB sind metasprachliche Hinweise in dieser Hinsicht sehr behilflich, die relativ häufig vorkommen und den phraseologischen Charakter eines Ausdrucks unter Beweis stellen, z. B.: besonders häufig in der Redensart; auch aus einer Fabel stammt die Redensart; also auch sprichw.; weitere Redensarten und Sprichwörter; sprichwörtlich; im 15. 16. Jahrh. begegnet öfters eine Redensart usw. Im SJP wurden die Phraseologismen viel seltener durch metasprachliche Hinweise markiert und eher unsystematisch verzeichnet, was die Exzerption noch zusätzlich erschwert.

Aufgrund der Tatsache, dass die Identifikation von Phraseologismen in älteren Wörterbüchern Schwierigkeiten bereitet und sowohl das SJP als auch das DWB in der Vorphase der Phraseologie-Forschung (vgl. Kühn 2007: 620) entstanden sind, entscheide ich mich für eine breit gefasste Definition des Terminus Phraseologismus, die nicht nur die Phraseologie im engeren Sinne (Phraseme/Idiome), sondern auch die Phraseologie im weiteren Sinne (Sprichwörter, Antisprichwörter, Sagwörter/Wellerismen, Lehnsprichwörter und geflügelte Worte) einschließt (vgl. Palm 1997: 1-6). Nach dieser Definition verfügt ein Phraseologismus über die folgenden Merkmale: Polylexikalität, Festigkeit/Stabilität und Idiomatizität (vgl. Burger 1998: 14-15; Lewicki 2003: 158), wobei die letzte Eigenschaft nicht obligatorisch ist.

\section{Die Wahl des phraseologischen Materials}

Als thematische Gruppe wurden Phraseologismen mit Tierkomponenten: kot/Katze und pies/Hund gewählt, getreu der Auffassung, dass Hund und Katze polar zusammengehören. Die Tierphraseologismen wurden nicht zufällig ausgewählt. Sowohl das Polnische als auch das Deutsche verfügt erstens über eine große Menge von festen Wortverbindungen, die mit Tieren verbundene Lexeme enthalten. Zweitens ist die Phraseologie mit Tierbezeichnungen als Konstituenten sehr bildhaft und anschaulich. Tiere als Bildspender "denotieren durch Metaphorisierungsprozesse zumeist Eigenschaften des Menschen, indem tierische Merkmale auf das menschliche Verhalten projiziert werden" (Chrissou 2000: 83). Angesichts dessen, dass zwischen Katzen und Hunden - zumindest auf der sprachlichen Ebene - eine angeborene Feindschaft besteht, erscheint das phraseologische Material um so interessanter. Aus dem SJP wurden insgesamt 30 Phraseologismen mit dem Lexem kot und 51 mit pies exzerpiert, aus dem DWB 63 Phraseologismen mit der Komponente Katze und 55 mit Hund. 
Das Ziel und die Methoden der Untersuchung

Das vorrangige Ziel der vorliegenden Untersuchung besteht darin, festzustellen, wie viele feste Wortverbindungen mit den Tierkomponenten kot/Katze und pies/Hund ausgestorben sind und dadurch als historische Phraseologismen angesehen werden können und wie viele noch heute in identischer oder ähnlicher Form gebräuchlich sind. Bei diesem Teil der Untersuchung sind polnische und deutsche phraseologische Wörterbücher behilflich, die als gegenwärtige Werke gelten, d. h. um/nach 2000 veröffentlicht wurden. Auch die Korpora Das Digitale Wörterbuch der deutschen Sprache (DWDS) ${ }^{1}$ und Narodowy Korpus Języka Polskiego (NKJP) ${ }^{2}$ - erlauben Vermutungen darüber anzustellen, ob eine Wortverbindung noch allgemein gebräuchlich ist oder nicht mehr verwendet wird. Ferner soll in diesem Beitrag dargestellt werden, welche Bedeutungen, Symbole und Bilder zusammen mit den als historisch zu bezeichnenden Phraseologismen verloren gegangen sind. Eine Antwort auf diese Frage geben in den meisten Fällen die beiden analysierten Wörterbücher selbst und wenn nicht, werden andere einschlägige Wörterbücher oder Werke hinzugezogen. Zum Schluss wird untersucht, ob die aus dem SJP und DWB exzerpierte polnische und deutsche Phraseologie mit den Tierlexemen kot/Katze und pies/Hund Ähnlichkeiten aufweist oder nicht, d. h. es soll herausgestellt werden, ob die Tierphraseologismen eher internationalen Charakter haben oder nur für eine spezielle Sprachgemeinschaft typisch sind.

\section{Ausgestorbene Phraseologismen mit den Komponenten kot/Katze und pies/Hund}

Meine Untersuchungen zeigen, dass 63\% der Phraseologismen mit der Komponente kot und $78 \%$ der Phraseologismen mit dem Lexem pies, die im SJP verzeichnet wurden, ausgestorben sind. Nur $37 \%$ der festen Wortverbindungen mit der Komponente kot und $22 \%$ mit pies können also als gebräuchlich bezeichnet werden. 83\% der Phraseologismen mit der Komponente Katze und 60\% der festen Wortverbindungen mit dem Element Hund gelten als historisch. Das bedeutet, dass 17\% der Phraseologismen mit dem Lexem Katze und 40\% mit Hund auch heute benutzt werden.

Die beiden folgenden Listen enthalten die im SJP und DWB erfassten Phraseologismen mit den Komponenten kot/Katze und pies/Hund. Die Rangfolge der Wortverbindungen in den Listen entspricht der Rangfolge in den Wörterbüchern. Die Nennform der Phraseologismen ist unverändert, nur die Rechtschreibung wurde auf den aktuellen Stand gebracht. Manchmal werden mehrere Varianten angegeben. Die historischen (d. h. ausgestorbenen, nicht mehr geläufigen) Phraseologismen wurden mit dem graphischen Zeichen " $\uparrow "$ und die aktuellen festen Wortverbindungen mit "- markiert. Es muss an dieser Stelle jedoch hinzugefügt werden, dass man auch diese Phraseologismen als aktuell betrachtet, die heute in einer etwas abgewandelten Form gebraucht werden (die heutige Form wird in den meisten Fällen in Fußnote angegeben).

\footnotetext{
1 Über die Webseite des DWDS hat man Zugang zu zwei Korpora: dem Kernkorpus des 20./21. Jahrhunderts, das Belege aus Texten ab 1900 bis 1999 enthält, und dem Zeit-Korpus, in dem die Pressetexte aller ZEITAusgaben von 1946-2009 vertreten sind. Um das aktuelle Vorkommen der aus dem DWB exzerpierten Phraseologismen zu überprüfen, wurden nur die Belege aus den Jahren 1989-2014 berücksichtigt. In den beiden Korpora wurde zwischen April und November 2014 recherchiert.

2 Auch im NKJP wurde in einem Zeitraum zwischen April und November 2014 recherchiert. Man berücksichtigte nur die Belege aus den Jahren 1989-2014.
} 
Liste der im SJP verzeichneten Phraseologismen mit den Komponenten kot und pies:

\section{kot}

Gdy kota myszy nie czują, bezpiecznie sobie harcują. ${ }^{3}$

Kot niełowny, chłop niemowny, często głodny. [...] Kot głodny, gdy niełowny, chłop gdy niedomowny. ${ }^{4}$

† Jako kot mówi, miarą. [...] Trzymaj miarę w wydatkach, miarą kotek mówi. [...] Miarą kocie, idzie o cię.

$\dagger$ Wierz mi, tu kot za uchem niejednemu $†$ Kto miłuje przyjaciela, miłuje i psa jego. wrzaśnie.

$\uparrow$ Jakie niebezpieczeństwa i jakie kłopoty miewa, komu już we łbie grzebią więc te koty!

Czatowałem na niego właśnie, jak kot na przepiórkę. 5

† On jako kot na mysz, na każdego godzi.

$\uparrow$ Kot na ledzie, [...] jako kot na gołoledzi.

$\dagger$ Gdzie nie masz kota, nie masz krotochwili.

$\uparrow$ Gdy kota głaszczą, mamroce.

$\dagger$ Im kota bardziej głaszczesz, tym bardziej ogon wznosi. [...] Głaszcz ty kotowi skórę, a on ogon w górę.

Im kot starszy, tym ogon twardszy.

$\uparrow$ Wstyd kota w ogon.

$\dagger$ Krajką go bił, a onego bolało, jakby go kot po ręku lizał.

† Dwaj być nie mogą w jednym worze koci bez zwady; zawsze na się mruczą, zawsze sapią. [...] Dwaj koci w jednym worze.

\section{pies}

Tyle dbam o to, jak pies o piątą nogę.

† Byś swemu psu i nogę uciął, przecie on za tobą pójdzie.

† Lepszy dobry pies, niż zły człowiek.

$\uparrow$ Pies dobry stoi za hajduka.

$†$ Gdzie wielkie stado, psów wiele trzeba.

† Psa zbiegłego z łańcucha łatwo poznać.

$\dagger$ Wiele psów zająca śmierć.

$\uparrow$ Co pies szczeknie.

Nietrudno o kij, kto chce psa uderzyć. ${ }^{6}$

$\dagger$ Uciekli, jak psi oparzeni.

$\dagger$ Pies na swych śmieciach śmielszy.

$\uparrow$ Pies gorszy, co milczkiem kąsa.

$\uparrow$ Kiedy pies śpi, a żyd przysięga, rzadko wierzyć trzeba.

† Psa nie drażnij.

\footnotetext{
${ }^{3}$ Die gebräuchliche Form des Phraseologismus ist: Jak kota nie ma, (to) myszy harcuja/biegaja/tańcuja (Müldner-Nieckowski 2004: 324). Ausgewählte Belege aus dem NKJP: Gdy kota nie ma, myszy harcuja; Myszy harcuja, gdy kota nie czuja.

${ }^{4}$ Chlop mowny a kot lowny z glodu nie zemra (PWN 2005: 37). NKJP: Chlop mowny, to kot towny; Kot musi być towny, a chtop mowny.

${ }^{5}$ Czatować na kogoś jak kot na mysz/wilk na owcę (Müldner-Nieckowski 2004: 324).

${ }^{6}$ Kto chce psa uderzyć, ten kij zawsze znajdzie (PWN 2005: 356). NKJP: Jak się chce psa uderzyć, kij zawsze się znajdzie; Jak się chce uderzyć psa, to kij się zawsze znajdzie.
} 
- Póki świat, światem, pies kotowi nie † Nie ciągnij psa za ogon, bo cię ukąsi. będzie bratem. ${ }^{7}$

Kochać kogo, jak psi kota. ${ }^{8}$

Każdy kot w nocy czarny (bury).

- Targował kota w worze. ${ }^{9}$

Dobrze mówią przy dworze, nie kupuj † Łaknąć jak pies.

kota w worze.

$\dagger$ Kota za lisa przedać.

$\dagger$ Nie mieć się na niedźwiedzia kocie † Psi go zjedli.

zagorzały, Tyś niewielkie stworzenie, a niedźwiedź niemały.

$\uparrow$ On stroi sobie apetyt na naszą Jejmość; ale ja go tak odsadzę, jak kota od mleka.

- Biega jak kot zagorzały. ${ }^{11}$

$\uparrow$ Kot jeszcze na ognisku.

† Kota paść.

$\dagger$ Kota ciągnąć.

$\uparrow$ Mam kota.

$\uparrow$ Nie dba o myszy, bo kota ułapi.

Koty albo lisy z kim drzeć.
† Pies zdechły już nie kąsa.

$\dagger$ Złego psa prędziej wilcy jedzą.

Żyją jak pies z kotem.

Jestem głodny jak pies.

Dobra psu mucha. ${ }^{10}$

- Nie rzucaj na psa sadła. ${ }^{12}$

$\uparrow$ Zjadł pies sadło.

† Psy wyją, a miesiąc świeci.

$\uparrow$ Pies szczeka, oszczerca kłamie.

$\uparrow$ Psu oczy przedał.

† I psy bywają w kościele.

† Jaki pies do kościoła, taki z kościoła.

† Wraca się do swojego nałogu, jak pies do swego zrzutu.

$\uparrow$ Daj to psu, co masz na sercu.

$\uparrow$ Nie da psa ni na kim.

$\uparrow$ Złajał mnie jak psa.

Jak psa, z domu go wypędził.

† Jak do psa palnę.

\footnotetext{
${ }^{7}$ NKJP: Jak świat światem, Polak Niemcowi nie będzie bratem; Jak świat światem, Niemiec dla Polaka nie będzie bratem.

${ }^{8}$ Kochać sięjak pies z kotem (PWN 2005: 356).

${ }^{9}$ Kupić kota $w$ worku (PWN 2005: 184). NKJP: [...] żeby nie kupować kota $w$ worku; [...] nie będą kupować kota w worku.

${ }^{10}$ Dobra psu i mucha (PWN 2005: 356). NKJP: Dobra psu i mucha, gdy sama wleci do brzucha; Dobra i psu mucha, kiedy głodny.

11 Biegaćllatac jak kot z pęcherzem (PWN 2005: 184).

12 Nie dla psa kietbasa, (nie dla kota sadto) (PWN 2005: 356). NKJP: Nie dla psa kiełbasa.
} 
Niewolnicy jak psy im służyli.

$\uparrow$ Za psa to stoi.

- Wszystko na psa pójdzie. ${ }^{13}$

† Brzydzę się nim bardziej, niż wężem i wściekłym psem. [...] Ona się tym, jak zgniłym psem brzydziła.

$\dagger$ Psy mu za uchem wyją.

† Umie swego psa leczyć.

$\dagger$ By na psie raz..

† Jak na psa łyko.

† Ostatek niechaj psi orzą.

† Psa za lisa przedawać.

$\dagger$ Kto za psy lega, za pchły wstaje.

$†$ Nie porwie pies, to co wilk puści.

Jechał cię pies. ${ }^{14}$

$\dagger$ Pies na robotę.

Ty psie!

Liste der im DWB verzeichneten Phraseologismen mit den Komponenten Katze und Hund:

\section{Katze}

$\uparrow$ Sich wie die Katze waschen. ${ }^{15}$

$\dagger$ Falsch wie eine Katze.

$\dagger$ Böse Katzen die vornen lecken, hinten kratzen.

Sie leben zusammen wie Hund und Katze.

$\uparrow$ Der Katzen Spiel ist der Mäuse Tod.

Mit einem spielen wie die Katze mit der (sterbenden) Maus. ${ }^{16}$

$\uparrow$ Die Katze spielt mit den Mäusen wenn sie satt ist.

- Die Katze lässt ihres Mausens nicht. ${ }^{17}$

\section{Hund}

$\uparrow$ Alte Hunde sind übel bändig zu machen.

Er ist bekannt wie ein bunter Hund.

$\uparrow$ Lasse inzwischen die Leute reden, und die Hunde bellen.

- Toter Hund beißt nicht.

$\uparrow$ Wann ein alter Hund baffet, so siehe aus.

$\uparrow$ Machst du dich zum Schaf, so beißen dich die Hunde.

$\dagger$ Es schadet nicht wann die Hunde schon bellen, wann sie nur nicht beißen.

怾 Hunde, die viel bellen, beißen nicht.

\footnotetext{
13 Ktoś/coś schodzi na psy (Müldner-Nieckowski 2004: 527).

14 Jebat to pies (Müldner-Nieckowski 2004: 527). NKJP: J... cię pies.

15 Das heute verwendete Kompositum Katzenwäsche ('kurzes, oberflächliches Sichwaschen') scheint an diesen Phraseologismus anzuknüpfen (DUDEN 1996: 822).

16 DWDS: Spielen wie die Katze mit der Maus.
} 
Der Katze die Schelle nicht anhägen wollen. ${ }^{18}$

Er klettert wie eine Katze.

Auf die Füsse fallen wie eine Katze.

$\dagger$ Katzen und Herren fallen immer auf die Füsse.

$\dagger$ Er hat ein Leben wie eine Katze, ein wahres Katzenleben.

Darum herum gehen wie die Katze um den heißen Brei. ${ }^{20}$

$\uparrow$ Dessen Namen noch keine Katze kennt, geschweige ein Mensch.

$\uparrow$ Sich keine Katze dünken lassen.

$\uparrow$ Darf doch die Katze den Kaiser ansehen?

$\uparrow$ Was weiß die Katze vom Sonntage? $\uparrow$ An fremden Hunden und Kindern ist das Brot verloren.

$\uparrow$ Da kräht weder Hund noch Hahn danach.

$\uparrow$ Dass ich bis an die Kitzing weder Hund noch Katze, viel weniger einen Menschen antreffen würde.

Man muss den schlafenden Hund nicht wecken. $^{19}$

$\dagger$ Der Hund ist tapfer auf seinem Mist.

- Weil es denn heute so abscheulich Wetter ist, dass man keinen Hund vor die Tür jagt.

Der doch mit all seinem Verstand kaum einen Hund könnte aus dem Ofen locken. ${ }^{21}$

- Viele Hund der Hasen Tod. ${ }^{22}$

$\uparrow$ Mit willigen Hunden fährt man bald.

$\dagger$ Hingegen aber ist ein junger Hund zum Jagen viel freudiger als ein alter Löw.

- Mit allen Hunden gehetzt sein.

$\dagger$ Wenn der Hund wacht, mag der Hirte schlafen.

$\dagger$ Wenn die Hunde schlafen, hat der Wolf gut Schafe stehlen.

$\dagger$ Die Katze isst wohl Fische, sie will sie aber nicht fangen.

$\dagger$ Man jagt die Katze zu spät vom Speck, Arbeiten müssen wie ein Hund.

\footnotetext{
17 Die Katze lässt das Mausen nicht (Duden 11 2002: 401). DWDS: Die Katze lässt das Mausen nicht.

18 Der Katze die Schelle umhängen (Duden 11 2002: 402). DWDS: [...] daß jemand der Katze die Schelle umhängt; [...] welcher der Katze die Schelle umhängt; Ich soll immer der Katze die Schelle anhängen.

19 Schlafende Hunde wecken (Duden 11 2002: 663). DWDS: Man solle nicht schlafende Hunde wecken; weil niemand schlafende Hunde wecken will.

${ }^{20} \mathrm{Um}$ etwas (herum)schleichen wie die Katze um den heißen Brei (Duden 11 2002: 347). DWDS: [...] herumschleichen wie die Katze um den heißen Brei; [...] schlich wie die Katze um den heißen Brei.

21 Mit etw. keinen Hund hinter dem Ofen hervorlocken (können)/vom Ofen locken (können) (Duden 112002 : 378). DWDS: [...] lockt keinen Hund hinter dem Ofen hervor; [...] möchte man keinen Hund hinter dem Ofen hervorlocken.

22 Viele Hunde sind des Hasen Tod (Duden 11 2002: 377). DWDS: Zu frühe Koalitionsaussagen sind des Hasen Tod.

23 DWDS: Das ist für die Katze!
} 
wenn er gefressen ist.

$\dagger$ Das ist der Katz den Käs anvertraut. † † †inem den Hund lesen.

$\uparrow$ Wenn man die Katze auf den Käse bindet, † Wer einen Hund will werfen, findet bald so frisst sie nicht. einen Prügel.

$\dagger$ Die Katz über den Käs kommen lassen.

Der Hund frisst wieder, was er gespien hat.

$\uparrow$ Die Katz ist hungrig, wenn sie ans Brot $\uparrow$ Neidisch wie ein Hund. geht.

$\uparrow$ Der Katze die den Spieß leckt, vertraut den Braten nicht.

$\uparrow$ Es ist zuviel von der Katze begehrt, dass sie bei der Milch sitze und nicht schlecke.

$\dagger$ Leichte Gesellen wollen sich an ihren Ehegatten nicht ersättigen lassen, sondern wärmen sich bei anderen Weibern, und halten ihre eheliche Pflichte wie der Hund die Fasten.

† Die Katze will auch Bratwürste.

$\uparrow$ Schwerlich essen die Hunde Bratwürste, sie stehlen sie denn.

$\uparrow$ Geschäftig wie eine Katze die sieben Töpfe † Den Hund nach Bratwürsten schicken.

zugleich zu lecken hat.

$\dagger$ Er weiß wo die Katze in Teig langt.

$\uparrow$ Der Hund hinkt an einem Bein.

Die Katze im Sack kaufen.

$\uparrow$ Den Hund hinken lassen.

$\uparrow$ Da ist der Katz gleich gestreut.

$\uparrow$ Hunde nach Bautzen führen.

$\uparrow$ Die Katze ist gern wo man sie strählt Auf den Hund kommen. (streichelt).

$\uparrow$ Er sei unter den Katzen gewesen.

Einen auf den Hund bringen.

$\uparrow$ Es gilt der Katze (um) den Schwanz.

Über den Hund kommen. ${ }^{24}$

$\uparrow$ Es müsse eine andere Katz im Heu sitzen.

Das wär das Rechte, dann kommst du vom Hund auf den Bettelsack.

$\dagger$ Davon gehen wie die Katze vom $\uparrow$ Meister geben ihren Gesellen den Hund, Taubenschlage.

[...] die Gesellen bekommen den Hund.

Bei Nacht sind alle Katzen grau.

$\dagger$ Einer ist so verachtet, dass nicht einmal ein Hund ein Stück Brot von ihm nimmt. [...] dass nicht ein Hund sich um ihn kümmert, sein gedenkt. [...] dass die Hunde ihn anpissen.

$\dagger[\ldots]$ die Katze weiß wohl, wem sie den Bart $†$ Er muss es haben als hätte ihn ein Hund

${ }^{24}$ Kommt man über den Hund, kommt man auch über den Schwanz (Duden 11 2002: 378). 
leckt.

$\dagger$ Vernaschte Katzen machen achtsame Etwas geht vor, für die Hunde, [...] für die Mägde.

$\uparrow$ Lässt sich die Katze streicheln, ist sie † Je magrer der Hund, je größer die Flöhe. schon mehr bei Leuten gewesen.

$\dagger$ Ließe die Katze das mausen, so bliebe der † Er schüttelt es ab, wie der Hund die Flöhe. Kater draußen.

$\uparrow$ Gestohlene Katzen mausen gern.

$\dagger$ Wer mit Katzen jagt, fängt gern Mäuse.

$\uparrow$ Es sind so gute Katzen die die Mäuse verjagen, als die sie fangen.

$\uparrow$ Gute Katzen mausen daheim und in andern Häusern.

$\dagger$ Will die Katze nicht mausen, so lässt sie draußen.

$\uparrow$ Üble Katze so nicht von selber maust.

$\uparrow$ Katze die mausen will, wird nie miauen.

$\uparrow$ Hat die Katze Junge, so lernt sie mausen.

Wenn die Katze aus dem Hause ist, - Du Hund!

springen die Mäuse über Stühl und Bänke. ${ }^{26}$

$\uparrow$ Bring eine Katze nach England, sie wird miauen. [...] man führe eine Katze in England, so wird sie doch miauen.

$\uparrow$ Reist eine Katze, so kommt ein Mäusfänger † Einen Hund ins Spiel werfen. wieder.

$\uparrow$ Gebrühte Katze scheut auch das kalte Wasser.

$\uparrow$ Grindige (unnütze, sterbende, versengte)

Katzen leben lange.

$\uparrow$ Tote Katzen beißen auch die Mäuse.

$\uparrow$ Wer sich mit Hunden niederlegt, steht mit Flöhen auf.

$\uparrow$ Der Hund reitet auf dem Arsche mit ihme.

Hunde bellen den Mond an. ${ }^{25}$

$\uparrow$ Der Hund geht mir vor dem Licht um.

$\dagger$ An kleinen Riemen lernen die Hunde Leder kauen.

$\dagger$ Es bekommt einem wie dem Hund das Gras.

Da liegt der Hund begraben.

安- Ein Hund sein.

Zwischen Hund und Wolf. 
$\dagger$ Wer Katzen gerne hat, bekommt eine

schöne Frau.

$\uparrow$ Verliebt wie eine Katze.

$\uparrow$ Die Katze heben.

$\uparrow$ Die Strebkatze ziehen.

$\dagger$ Er muss die Katz durch den Bach ziehen, ausessen was er nicht einbrockte.

\section{Historische Phraseologismen mit der Komponente kot/Katze}

Über die Katze schrieben Jacob und Wilhelm Grimm: "[...] eins der merkwürdigsten und fragenreichsten Wörter". Davon, dass sich die Lexikographen nicht geirrt haben und dass die Symbolik der Katze äußerst mannigfaltig ist, zeugen die im SJP und DWB verzeichneten Phraseologismen, die heute als historische Wortverbindungen angesehen werden.

\subsection{Freundschaft und Hilfsbereitschaft}

Die Katze gilt erstens als die Mäuse- und Rattenfängerin und dadurch als Beschützerin der Ernte und Helferin des Menschen, was nicht nur in der gegenwärtigen Phraseologie des Polnischen und des Deutschen ihren Ausdruck fand (vgl. das polnische Sprichwort Gdy kota myszy nie czuja, bezpiecznie sobie harcuja und seine deutsche Entsprechung Wenn die Katze aus dem Hause ist, springen die Mäuse über Stühl und Bänke), sondern auch in den historischen Phraseologismen: On jako kot na mysz, na każdego godzi (wörtl. 'Wie die Katze auf die Maus zielen'); Nie dba o myszy, kto kota ułapi (wörtl. 'Wer die Katze gefangen hat, macht sich keine Sorgen um die Mäuse'); Der Katzen Spiel ist der Mäuse Tod. ${ }^{27}$

Als Helferin und Liebling des Menschen wird die Katze oft gestreichelt: Głaszcz ty kotowi skórę, a on ogon $w$ górę (SJP: 'jd. wird hochmütig' ${ }^{28}$ ); Die Katze ist gern, wo man sie strählt/streichelt (DWB: 'man hört gern sich loben'). ${ }^{29}$ Mit dem Schwanz ist auch ein anderer deutscher Phraseologismus verbunden: Es gilt der Katze (um) den Schwanz, der im DWB als 'es geht hart an Leib' erklärt wurde. Die Katze zeigt eine enge Bindung zu ihrem Besitzer, indem sie mit ihm spielt, sich an ihn schmiegt und ihn leckt: Gdzie nie masz kota, nie masz krotochwili (wörtl. 'Wo es keine Katze gibt, gibt es keinen Spaß'); Krajka go bit, a onego bolało, jakby go kot po ręku lizat (wörtl. *Etwas tut weh, als ob die Katze jemanden lecken würde); Die Katze weiß wohl, wem sie den Bart leckt (der Aphorismus von Goethe wird im

\footnotetext{
27 Andere deutsche Beispiele: Die Katze spielt mit den Mäusen, wenn sie satt ist; Ließe die Katze das mausen, so bliebe der Kater draußen; Gestohlene Katzen mausen gern; Wer mit Katzen jagt, fängt gern Mäuse; Es sind so gute Katzen die die Mäuse verjagen, als die sie fangen; Gute Katzen mausen daheim und in andern Häusern; Will die Katze nicht mausen, so lässt sie draußen; Üble Katze so nicht von selber maust; Katze die mausen will, wird nie miauen; Hat die Katze Junge, so lernt sie mausen; Reist eine Katze, so kommt ein Mäusfänger wieder; Tote Katzen beißen auch die Mäuse.

28 Übersetzung der Autorin (D.J.); auch in allen anderen Erklärungen aus dem SJP.

${ }^{29}$ Andere Beispiele: Gdy kota głaszcza, mamroce (wörtl. 'Wenn die Katze gestreichelt wird, schnurrt sie'); Im kota bardziej głaszczesz, tym bardziej ogon wznosi (wörtl. 'Je mehr die Katze gestreichelt wird, desto höher hebt sie ihren Schwanz'); Lässt sich die Katze streicheln, ist sie schon mehr bei Leuten gewesen.
}

ISSN 1615-3014 
DWB so erklärt: 'Liebe will Lohn'). Die Katze reagiert aber nicht, wenn der Besitzer ihr einen Tadel erteilt: Wstyd kota w ogon (im SJP als 'nie dba o strofowanie' erklärt).

\section{$5.2 \quad$ (Hunde)Feindlichkeit}

Die Feindseligkeit von Hund und Katze liegt dem populären polnischen und deutschen Phraseologismus zugrunde: żyć jak pies z kotem/wie Hund und Katze zusammenleben. Im SJP kann man auch eine andere Wortverbindung finden, wo die Katze mit dem Hund korreliert: Póki świat, światem, pies kotowi nie będzie bratem (wörtl. 'Die Katze wird nie zum Bruder des Hundes'). Nicht nur ein Hund, sondern auch eine andere Katze kann zur Gegnerin werden: Dwaj być nie moga w jednym worze koci bez zwady; zawsze na się mrucza, zawsze sapia; [...] Dwaj koci w jednym worze (im SJP folgendermaßen erklärt: 'trudna zgoda, ogień woda' - es ist nicht möglich, dass zwei Katzen friedlich miteinander leben), unter den Katzen gewesen sein (DWB: 'von einem der zerzaust, zerkratzt kommt').

\subsection{Geräusche}

Die Geräusche, die für jede Katze charakteristisch sind, bilden die Grundlage für einige polnische Phraseologismen: Jako kot mówi, miara. [...] Trzymaj miarę w wydatkach, miara kotek mówi. [...] Miara kocie, idzie o cię (das Wort miau wurde mit dem polnischen Lexem miara ('Maß') assoziiert, daher: miauen als 'Maß halten'); Kot za uchem niejednemu wrzaśnie (es geht hier um Katzengejaule, das depressiv wirkt).

\subsection{Trinken und Essen}

Die Tatsache, dass die Katze gern Milch trinkt, findet ihren Ausdruck in polnischen und deutschen historischen Phraseologismen: On stroi sobie apetyt na nasza Jejmość; ale ja go tak odsadze, jak kota od mleka (wörtl. 'Jdn. wie die Katze von der Milch trennen'); Es ist zuviel von der Katze begehrt, dass sie bei der Milch sitze und nicht schlecke; Geschäftig wie eine Katze die sieben Töpfe zugleich zu lecken hat. Auch die Speisen, die die Katze gern oder ungern hat, werden zu Komponenten einiger deutscher Wortverbindungen: Die Katz ist hungrig, wenn sie ans Brot geht; Der Katze die den Spieß leckt, vertraut den Braten nicht. ${ }^{30}$ Der polnische Phraseologismus Kot jeszcze na ognisku (wörtl. 'Die Katze ist noch auf dem Feuer') wurde im SJP als 'das Mittagessen ist noch nicht fertig' erklärt. ${ }^{31}$

\subsection{Wasserfeindlichkeit}

Der historischen Phraseologie nach geht die Katze dem Wasser aus dem Wege: Sich wie die Katze waschen ('ungern'); Die Katze isst wohl Fische, sie will sie aber nicht fangen; Gebrühte Katze scheut auch das kalte Wasser. Auch gefrorenes Wasser ist für die Katze gefährlich: Kot na ledzie, [...] Jako kot na gololedzi (SJP: 'eine gefährliche Situation'). Im deutschen

\footnotetext{
30 Andere Beispiele: Die Katze will auch Bratwürste; Er weiß wo die Katze in Teig langt (DWB: 'ist listig'); Das ist der Katz den Käs anvertraut; Wenn man die Katze auf den Käse bindet, so frisst sie nicht; Die Katz über den Käs kommen lassen; Was weiß die Katze vom Sonntage? (DWB: 'Für sie ist zum Futter das Schlechteste gut genug, die Reste des Mahls').

31 Die Herkunft dieser Wendung ist schwer zu erklären. Vielleicht geht es darum, dass die Katze auf dem Herd liegt, so dass nicht gekocht wird (vgl. Anusiewicz 1990: 122).
} 
Phraseologismus Da ist der Katz gleich gestreut (DWB: 'der Sache gleich abgeholfen') wird auf die geschwinde Beseitigung von Katzendreck durch Bestreuen mit Sand (und nicht mit Wasser!) hingewiesen.

\subsection{Liebe und Geld}

Die Katze wurde auch mit Liebe assoziiert: Jakie niebezpieczeństwa i jakie kłopoty miewa, komu już we tbie grzebia więc te koty! (SJP: 'Liebschaften') (wörtl. 'Jd. hat die Katzen im Kopf' - 'Jd. ist verliebt'); Wer Katzen gerne hat, bekommt eine schöne Frau; Verliebt wie eine Katze. Im Polnischen bedeutete die Katze auch 'Geldbeutel', daher mam kota ${ }^{32}$ als 'pieniężny jestem' (wörtl. 'Ich habe die Katze' - 'Ich habe Geld').

\subsection{Schnelligkeit, Gewandtheit, Klugheit, Unabhängigkeit, Faulenzerei, Langlebigkeit und Falschheit}

Die Katze gilt als Symbol der Schnelligkeit, Gewandtheit, Klugheit, Unabhängigkeit, Faulenzerei und Langlebigkeit: Die Katz läuft im den Ruggen auf (DWB: 'Er hat wohl zu schaffen gewonnen'); Es läuft ihm die Katze den Buckel hinauf; Katzen und Herren fallen immer auf die Füsse; Ein Leben wie eine Katze haben; Davon gehen wie die Katze vom Taubenschlage (DWB: 'sich nach vollbrachtem Schaden ungesehen fortschleichen'); Es müsse eine andere Katz im Heu sitzen; Grindige (unnütze, sterbende, versengte) Katzen leben lange. Die deutschen historischen Phraseologismen, z. B. falsch wie eine Katze; Böse Katzen die vornen lecken, hinten kratzen zeugen davon, dass die Katze auch Falschheit symbolisierte.

\subsection{Minderwertigkeit}

Die Katze wird als etwas Minderwertiges betrachtet: Kota za lisa przedać (wörtl. 'Die Katze als den Fuchs verkaufen'); Nie miec się na niedźwiedzia kocie zagorzały, Tyś niewielkie stworzenie, a niedźwiedź niemały (wörtl. 'Die Katze soll sich nicht gegen den Bären auflehnen'); Dessen Namen noch keine Katze kennt, geschweige ein Mensch; Sich keine Katze dünken lassen (DWB: 'sich für was rechts halten').

\subsection{Alte Strafen}

Zwei polnische Phraseologismen mit der Komponente kot beziehen sich auf alte Strafen der Soldaten: kota ciagnać (wörtl. 'die Katze ziehen') im Sinne 'ausgelacht werden' und kota paść (wörtl. 'die Katze weiden') - 'geprügelt werden' oder 'im Gefängnis sitzen'.

\section{Historische Phraseologismen mit der Komponente pies/Hund}

Der Hund schwankt im symbolischen Bereich zwischen zwei Extremen - von positiver Bedeutung bis zur negativen, wobei in der historischen Phraseologie vor allem diese zweite Tendenz ihren Ausdruck fand.

\footnotetext{
32 Heute hat der Phraseologismus mieć kota (wörtl. *die Katze haben) eine andere Bedeutung 'być niezrównoważonym psychicznie, robić coś niezgodnie z oczekiwaniami' ('verrückt sein') (Müldner-Nieckowski 2004: 325).
} 
kot/Katze und pies / Hund im "Słownik języka polskiego" und im "Deutschen Wörterbuch"

\subsection{Treue, Freundschaft und Hilfsbereitschaft}

Als Symbol der Treue und Freundschaft kommt der Hund nur in einigen polnischen Phraseologismen vor: Byś swemu psu i nogę uciąt, przecie on za toba pójdzie (wörtl. 'Sogar wenn du deinem Hund das Bein abschneiden würdest, würde er dir nachgehen'); Lepszy dobry pies, niż zly człowiek (wörtl. 'Ein guter Hund ist besser als ein schlechter Mensch'). ${ }^{33}$ Die Rolle des Hundes als Helfer und Begleiter des Menschen beim Hüten der Schafe und Jagen drückt sich in den folgenden Phraseologismen aus: Gdzie wielkie stado, psów wiele trzeba (wörtl. 'Wo es eine große Herde gibt, braucht man viele Hunde'); Wenn der Hund wacht, mag der Hirte schlafen. ${ }^{34}$

\subsection{Untreue, Falschheit und Neid}

So wie Treue kann der Hund auch Untreue sowie Falschheit und Neid symbolisieren: Kiedy pies śpi, a żyd przysięga, rzadko wierzyć trzeba (wörtl. 'Man soll nicht glauben, wenn der Hund schläft und der Jude schwört'); Psi go zjedli (wörtl. 'Die Hunde haben ihn gegessen' SJP: 'Die Diener haben ihn gegessen'); An fremden Hunden und Kindern ist das Brot verloren; Neidisch wie ein Hund. ${ }^{35}$ Als interessant gilt auch der polnische Phraseologismus Pies na robote (wörtl. 'Hund auf Arbeit'), im SJP als 'faul' erklärt, besonders deswegen, dass heute die Wortverbindung ktoś jest pies na coś/na kogoś (PWN 2005: 355) vor allem mit der Vorliebe für etwas assoziiert wird.

\subsection{Boshaftigkeit und Aggressivität}

Der Hund wurde auch mit Boshaftigkeit und Aggressivität assoziiert: Wiele psów zajaca śmierć (die deutsche Entsprechung: Viele Hunde sind des Hasen Tod ist auch heute geläufig); Pies gorszy, co milczkiem kąsa (wörtl. 'Dieser Hund ist böser, der stillschweigend beißt'); Der Hund ist tapfer auf seinem Mist; Es schadet nicht wann die Hunde schon bellen, wann sie nur nicht beißen. ${ }^{36}$

\footnotetext{
${ }^{33}$ Andere Beispiele: Pies dobry stoi za hajduka (wörtl. *Ein guter Hund ist besser als der Heiduck); Kto mituje przyjaciela, mituje i psa jego (wörtl. 'Wer seinen Freund liebt, liebt auch seinen Hund'); Umie swego psa leczyć (SJP: 'den entstandenen Schaden wieder gutmachen').

${ }^{34}$ Andere Beispiele: Złego psa prędziej wilcy jedzq (wörtl. 'Ein böser Hund wird schneller von Wölfen gefressen'); Nie porwie pies, to co wilk puści (wörtl. 'Der Hund nimmt es nicht, was der Wolf gibt'); Wenn die Hunde schlafen, hat der Wolf gut Schafe stehlen; Hingegen aber ist ein junger Hund zum Jagen viel freudiger als ein alter Löw; Der Hund reitet auf dem Arsche mit ihme (DWB: 'wenn der Hund nicht lustig ist zu Jagen, so reit er auf dem Arsch').

${ }^{35}$ Andere polnische Beispiele: Pies szczeka, oszczerca ktamie (wörtl. 'Der Hund bellt, der Verleumder lügt'); Psu oczy przedat (so nennt man im SJP einen frechen Menschen); I psy bywaja w kościele (wörtl. 'Auch die Hunde gehen zur Kirche'); Jaki pies do kościoła, taki z kościoła; Daj to psu, co masz na sercu (SJP: 'Du bist ein falscher Mensch').

${ }^{36}$ Andere Beispiele: Psa nie drażij (SJP: 'Wecke keine schlafenden Hunde'); Nie ciagnij psa za ogon, bo cie ukąsi (wörtl. 'Ziehe den Hund am Schwanz nicht, da er dich beißt'); Pies zdechly już nie kasa (auch diese deutsche Entsprechung: Toter Hund beißt nicht ist in den gegenwärtigen Wörterbüchern präsent); Pies na swych śmieciach śmielszy (wörtl. 'Der Hund ist tapferer auf seinem Mist'); Mit willigen Hunden fährt man bald; Wann ein alter Hund baffet, so siehe aus; Machst du dich zum Schaf, so beißen dich die Hunde.
} 


\subsection{Minderwertigkeit}

Bei vielen polnischen und deutschen historischen Phraseologismen mit der Komponente pies/Hund erkennt man die Bedeutung der Minderwertigkeit und Verächtlichkeit: Za psa to stoi (SJP: 'Das ist nichts wert'); By na psie raz ('Jd. ist nicht ehrenhaft, gleichgütig gegen Beleidigungen'); Da kräht weder Hund noch Hahn danach; Dass ich bis an die Kitzing weder Hund noch Katze, viel weniger einen Menschen antreffen würde. ${ }^{37}$ Als interessant gelten in diesem Kontext zwei deutsche Idiome, die heute nicht mehr bekannt sind: Meister geben ihren Gesellen den Hund ('entlassen sie'); Die Gesellen bekommen den Hund ('werden entlassen').

\subsection{Hunger und Elend}

Davon, dass der Hund ein mühevolles Leben hat, oft hungrig ist, geprügelt und gebändigt wird, zeugen die folgenden Wortverbindungen: Laknać jak pies (wörtl. 'Appetit haben wie ein Hund'); Złajat mnie jak psa (wörtl. 'jmdn. wie einen Hund ausschimpfen'); Wer einen Hund will werfen, findet bald einen Prügel (die polnische Entsprechung Nietrudno o kij, kto chce psa uderzyć wird auch heute noch benutzt); Schwerlich essen die Hunde Bratwürste, sie stehlen sie denn. ${ }^{38}$ Mit des Hundes Elend werden auch Flöhe assoziiert, wovon die folgenden Phraseologismen zeugen: das polnische Sprichwort Kto za psy lega, za pchty wstaje und seine deutsche Entsprechung Wer sich mit Hunden niederlegt, steht mit Flöhen sowie Je magrer der Hund, je größer die Flöhe und Er schüttelt es ab, wie der Hund die Flöhe.

\subsection{Geräusche}

Auch des Hundes Bellen und Jaulen fanden ihren Ausdruck in der polnischen und deutschen Phraseologie: Co pies szczeknie ('bald'); Psy wyja, a miesiac świeci ${ }^{39}$; Psy mu za uchem wyja (vgl. oben Kot za uchem niejednemu wrzaśnie); Lasse inzwischen die Leute reden, und die Hunde bellen.

\footnotetext{
${ }^{37}$ Andere Beispiele: Nie da psa ni na kim (SJP: 'jd., der ständig stichelt'); Brzydzę się nim bardziej, niż wężem i wściektym psem (wörtl. 'sich vor jmdm. wie vor dem tollwütigen Hund ekeln'); Psa za lisa przedawać (vgl. oben Kota za lisa przedać); Ostatek niechaj psi orza; DWB: Einer ist so verachtet, dass nicht einmal ein Hund ein Stück Brot von ihm nimmt. [...] dass nicht ein Hund sich um ihn kümmert, sein gedenkt. [...] dass die Hunde ihn anpissen; Einem den Hund lesen ('wie einen Hund ausschimpfen oder behandeln'); Hunde nach Bautzen führen (DWB: 'in Verachtung und Ungemach sein').

38 Andere Beispiele: Psa zbiegłego z łańcucha łatwo poznać (wörtl. 'Es ist einfach, einen Hund zu erkennen, der sich von der Kette gerissen hat'); Zjadt pies sadło (SJP: 'jd. hat Gewissensbisse'); Wraca się do swojego nałogu, jak pies do swego zrzutu (wörtl. 'Jd. kehrt zu seiner Sucht zurück, wie der Hund dazu, was ihm hingeworfen wurde'); Jak na psa tyko (SJP: 'Jd. wird nur leicht geschlagen, so dass er nichts fühlt'; vgl. auch oben Krajka go bit, a onego bolato, jakby go kot po ręku lizat); Jak do psa palnę (wörtl. 'wie auf den Hund schießen'); Alte Hunde sind übel bändig zu machen.

${ }^{39}$ Vgl. pl. Pies szczeka/psy szczekaja, karawana idzie dalej oder dt. Die Hunde bellen, und/aber die Karawane zieht weiter (Duden 11 2002: 377).
} 


\section{$7 \quad$ Polnische und deutsche Phraseologismen mit den Komponenten kot/Katze und pies/Hund im Kontrast}

Die durchgeführte Analyse lässt den Schluss zu, dass die polnische und deutsche Phraseologie mit den Komponenten kot/Katze und pies/Hund vor allem in Bezug auf Symbolik und Semantik zahlreiche Ähnlichkeiten aufweist. Wenn es sich aber um Phraseologismen handelt, die als phraseologische Entsprechungen (Voll- oder mindestens Teiläquivalente) ${ }^{40}$ fungieren könnten, gibt es im analysierten Sprachmaterial nur einige Beispiele (vgl. die Tabelle 1 unten).

\begin{tabular}{|l|l|}
\hline \multicolumn{1}{|c|}{ SJP } & \multicolumn{1}{|c|}{ DWB } \\
\hline Żyją jak pies z kotem. & Sie leben zusammen wie Hund und Katze. \\
\hline Nie kupuj kota w worze & Die Katze im Sack kaufen. \\
\hline Gdy kota głaszczą, mamroce. & $\begin{array}{l}\text { Die Katze ist gern wo ma sie } \\
\text { strählt/streichelt. }\end{array}$ \\
\hline Każdy kot w nocy czarny (bury). & Bei Nacht sind alle Katzen grau. \\
\hline $\begin{array}{l}\text { Gdy kota myszy nie czują, bezpiecznie } \\
\text { sobie harcują. }\end{array}$ & $\begin{array}{l}\text { Wenn die Katze aus dem Hause ist, springen } \\
\text { die Mäuse über Stühl und Bänke. }\end{array}$ \\
\hline Psa nie drażnij. & $\begin{array}{l}\text { Man muss den schlafenden Hund nicht } \\
\text { wecken. }\end{array}$ \\
\hline Pies na swych śmieciach śmielszy. & Der Hund ist tapfer auf seinem Mist. \\
\hline Wiele psów zająca śmierć. & Viele Hund der Hasen Tod. \\
\hline Nietrudno o kij, kto chce psa uderzyć. & $\begin{array}{l}\text { Wer einen Hund will werfen, findet bald } \\
\text { einen Prügel. }\end{array}$ \\
\hline Kto za psy lega, za pchły wstaje. & $\begin{array}{l}\text { Wer sich mit Hunden niederlegt, steht mit } \\
\text { Flöhen auf. }\end{array}$ \\
\hline Ty psie! & \begin{tabular}{l} 
Du Hund! \\
\hline
\end{tabular} \\
\hline
\end{tabular}

Tabelle 1: Phraseologische Entsprechungen im SJP und DWB

\section{Schlussfolgerungen}

Aus dem Dargestellten ergeben sich einige Schlussfolgerungen:

1. Die deutsche und polnische Phraseologie mit den Elementen kot/Katze und pies/Hund weist eine relativ große 'Empfindlichkeit gegen Zeitablauf' auf. Die meisten Phraseologismen mit den ausgewählten Tierkomponenten sind ausgestorben und gelten heute als historisch (63\% der festen Wortverbindungen mit der Komponente kot und 78\% mit pies sowie $83 \%$ der Phraseologismen mit dem Element Katze und 60\% mit Hund). Außerdem werden viele als aktuell markierte Wortverbindungen heute in einer etwas abgewandelten Form verwendet.

\footnotetext{
${ }^{40}$ Die Einteilung der Entsprechungen in phraseologische Entsprechungen (auch Äquivalente genannt) und nichtphraseologische Entsprechungen erfolgt nach Worbs (1994: 165, 171).
} 
2. Die als historisch bezeichneten polnischen und deutschen Phraseologismen haben viele Ähnlichkeiten miteinander, wenn es sich um ihre Bildhaftigkeit, Symbolik und Semantik handelt. Viele Assoziationen und Bedeutungen sind auch heute noch aktuell, nur einige sind verloren gegangen.

3. Obwohl die polnische und deutsche Phraseologie mit den Lexemen kot/Katze und pies/Hund meistens auf den gleichen Metaphern basiert, sind im untersuchten Sprachmaterial nur wenige phraseologische Entsprechungen zu finden.

\section{Literatur}

Anusiewicz, Janusz (1990): "Językowo-kulturowy obraz kota w polszczyźnie". Etnolingwistyka 3: 95-138.

Burger, Harald (1998): Phraseologie. Eine Einführung am Beispiel des Deutschen. Berlin: Erich Schmidt.

Burger, Harald/Buhofer, Annelies/Sialm, Ambros (1982): Handbuch der Phraseologie. Berlin/New York: de Gruyter.

Chrissou, Marios (2000): Kontrastive Untersuchungen zu deutschen und neugriechischen Phraseologismen mit animalistischer Lexik. Essen: Clemon.

Das Digitale Wörterbuch der deutschen Sprache. www.dwds.de/ [04.11.2014].

Dräger Marcel (2009): "Auf der Suche nach historischen Phrasemen - oder: Wörterbücher als Korpora". Linguistik online 39/3: 33-43.

Duden (1996): Deutsches Universalwörterbuch. Mannheim: Duden.

Duden (2002): Redewendungen. Wörterbuch der deutschen Idiomatik 11. Mannheim: Duden. Grimm, Jacob/Grimm, Wilhelm (1854/1960): Deutsches Wörterbuch. Leipzig: Hirzel.

Kłosińska, Anna/Sobol, Elżbieta/Stankiewicz, Anna (2005): Wielki słownik frazeologiczny PWN z przysłowiami. Warszawa: PWN.

Kühn, Peter (2007): "Phraseologie des Deutschen: Zur Forschungsgeschichte". In: Burger, Harald/Dobrovol'skij, Dmitrij/Kühn, Peter/Norrick, Neal R. (ed.) (2007): Phraseologie. Ein internationales Handbuch der zeitgenössischen Forschung. Berlin/New York: de Gruyter: 619-643. (= Handbücher zur Sprach- und Kommunikationswissenschaft Band 28.2).

Lewicki, Andrzej Maria (2003): Studia z teorii frazeologii. Łask: Oficyna Wydawnicza LEKSEM.

Linde, Samuel Bogumił (1807/1814): Słownik języka polskiego. Warszawa: Drukarnia XX. Pijarów.

Matuszczyk, Bożena (2006): Słownik języka polskiego S.B. Lindego. Warsztat leksykografa. Lublin: Wydawnictwo KUL.

Müldner-Nieckowski, Piotr (2004): Wielki słownik frazeologiczny języka polskiego: wyrażenia, zwroty, frazy. Warszawa: Świat Ksiażki.

Narodowy Korpus Języka Polskiego. http://nkjp.pl/ [04.11.2014].

Palm, Christine (1997): Phraseologie. Eine Einführung. Tübingen: Narr.

Pepłowski, Franciszek (1970): "Samuel Bogumił Linde. W dwusetną rocznicę urodzin". Rocznik Kulturalny Kujaw i Pomorza 4: 99-110.

Röhrich, Lutz (2000): Lexikon der sprichwörtlichen Redensarten 4. Berlin: Digitale Bibliothek. 
kot/Katze und pies / Hund im "Słownik języka polskiego" und im "Deutschen Wörterbuch"

Sadiku, Milote (2011): "Deutsche und albanische Phraseologismen mit Tierbezeichnungen im Kontrast". Linguistik online 47/3: 141-152.

Schlaefer, Michael (2002): Lexikologie und Lexikographie. Eine Einführung am Beispiel deutscher Wörterbücher. Berlin: Schmidt.

Walter, Harry (2011): "Hüte dich vor bösen Katzen, die vornen lecken und hinten kratzen! Deutsche Redewendungen mit der Komponente "Katze" und ihre slawischen Äquivalente". Anuari de Filologia 1: 83-98.

Worbs, Erika (1994): Theorie und Praxis der slawisch-deutschen Phraseographie. Mainz: Liber. 